\title{
Performance of Receive Diversity UWB Systems with Pulse Amplitude and Position Modulation
}

\author{
Sangchoon Kim, Member, KIMICS
}

\begin{abstract}
In this paper, we extend ultra-wideband (UWB) single input single output (SISO) systems with a hybrid pulse amplitude and position modulation (PAPM) to single input multiple output (SIMO) systems using receive antenna diversity. The performance of a rake receive diversity combining scheme for UWB SIMO systems with a PAPM is examined in a log-normal multipath fading channel and also compared with that of a time-switched transmit diversity (TSTD) multiple input single output (MISO) system. It is seen that as the number of receive antennas increases, the receive diversity combining system improves the error performance. It is shown that the TSTD UWB MISO systems offer the performance equivalent to the receive diversity combining scheme for SIMO systems.
\end{abstract}

Index Terms - Ultra-Wideband (UWB), Single Input Multiple Output (SIMO), Pulse Amplitude and Position Modulation (PAPM), Time-Switched Transmit Diversity (TSTD)

\section{INTRODUCTION}

Ultra-wideband (UWB) radio technology has recently attracted considerable attention for indoor, short distance wireless communication systems with high data rate transmission [1]. In [2], the error performance of a rake receiver in a UWB single input single output (SISO) system with a pulse amplitude pulse modulation (PAPM) has been analyzed. Multiple antenna technique can be employed to enhance the performance through spatial antenna diversity in UWB communication systems. In dense multipath environments, multiple receive antennas combined with the rake receiver can be used to exploit spatial and temporal diversities in order to reduce the effects of multipath fading. In [3], the bit error rate (BER) performance of time hopping pulse position modulation (PPM) UWB systems with antenna array has been analyzed in the presence of multipaths. In [4], the performance of PPM UWB systems using multiple transmit and receive antennas has been investigated.

In this paper, a rake receive diversity combining scheme for UWB single input multiple output (SIMO)

\footnotetext{
Manuscript received August 10, 2010; revised September 15, 2010; accepted September 20, 2010.

Sangchoon Kim is with the Department of Electronics Engineering, Dong-A University, Busan, Korea (Email: sckim@dau.ac.kr)
}

systems with PAPM is considered as an extension of UWB SISO systems with PAPM presented in [2]. It consists of rake receivers with multiple parallel correlators. The BER performance of a linear receive rake diversity scheme is evaluated in a log-normal multipath fading channel and also compared with that of a timeswitched transmit diversity (TSTD) approach [4]. It is shown that as the number of receive antennas increases, the error performance is improved. It is seen that the TSTD UWB multiple input single output (MISO) systems achieve the same performance as the receive diversity combining SIMO systems while making the mobile units simple.

\section{RECEIVE DIVERSITY COMBINING APPROACH}

Consider a receive diversity combining approach for UWB SIMO systems. In pulsed UWB SIMO systems with binary PAPM, the transmitted signal from the transmit antenna has the form [2]

$$
x(t)=\sum_{q=-\infty}^{\infty} \sqrt{E_{x}} b_{0, q} w\left(t-q T_{s}-\frac{1-b_{1, q}}{2} \varepsilon\right)
$$

where $w(t)$ is the unit energy UWB pulse of short duration $T_{p}, E_{x}$ is the symbol energy, $T_{s}\left(>>T_{p}\right)$ is a frame duration. The $q$ th transmitted symbol is represented by two bits, $\left\{b_{0, q}, b_{1, q}\right\} \in\{11,1-1,-11,-1-1\}$. The symbols are precoded by interchanging $\{-11\}$ and $\{-1-1\}$. If $b_{1, q}=-1$, the pulse position is shifted relative to the time reference by $\varepsilon$. The signaling rate is assumed to be such that the received signal energy of a particular symbol is contained within one symbol time interval. Thus, since there is no intersymbol interference (ISI), a particular symbol interval in the receive modeling can be focused on. The signal, $x(t)$, transmitted from the transmit antenna goes through a multipath log-normal fading channel. The impulse response of the channel to the $m$ th receive antenna propagated from the transmit antenna is given by [5]

$$
h_{m}(t)=\sum_{l=0}^{L_{t}-1} \beta_{m}(l) \delta\left(t-l T_{p}\right)
$$


where $L_{t}$ is the total number of resolvable multipath components, $\delta(t)$ is the Dirac delta function, $T_{p}$ is the minimum path resolution, and $\beta_{m}(l)$ is the channel fading coefficient of the $l$ th resolvable path signal from the transmit antenna to the $m$ th receive antenna whose magnitude has a log-normal distribution. In order to consider a random phase inversion due to reflections, the channel gain $\beta_{m}(l)$ can be modeled as $\beta_{m}(l)=$ $\theta_{m}(l) \alpha_{m}(l)$ where $\alpha_{m}(l)$ is the log-normal fading magnitude term and $\theta_{m}(l) \in\{+1,-1\}$ indicates the pulse inversion with equal probabilty. The multipath intensity profile model for an indoor UWB channel is exponentially expressed by $E\left\{\left|\beta_{m}(l)\right|^{2}\right\}=e^{-\rho l}$ where $\rho$ is the power decay factor.

To maintain reasonable system complexity for signal detection, the receiver in Figs. 1 and 2 is assumed to exploit only a subset of $L\left(\leq L_{t}\right)$ resolvable paths. The received signal $r_{m}(t)$ at the $m$ th receive antenna, $m=1,2, \cdots, M$, is a sum of signals from the transmit antenna written as

$$
r_{m}(t)=\sum_{l=0}^{L-1} \beta_{m}(l) x\left(t-l T_{p}\right)+\eta_{m}(t)
$$

where $\eta_{m}(t)$ is a real zero-mean white Gaussian noise process with a two-sided power spectral density of $N_{0} / 2$. Assuming perfect estimates of multipath delays, the receiver uses two template waveforms

$$
\phi^{(i)}(t)=w(t-i \varepsilon), \quad i=0,1
$$

It is also assumed that perfect estimates of the channel coefficients are available to the receiver. The correlator output at each receiver branch is integrated for the $L$ resolvable paths and then combined using maximal ratio combining (MRC). As shown in Fig. 2, the resulting output at the $m$ th antenna's rake receiver branch are expressed as

$$
y_{m}^{(i)}=\sum_{l=0}^{L-1} \beta_{m}(l) \int_{0}^{T_{s}} r_{m}(t) \phi^{(i)}\left(t-l T_{p}\right) d t
$$

We assume in this work that an orthogonal signaling scheme [2] is used. Thus we select the minimum value of $\varepsilon$ to satisfy $\int_{-\infty}^{\infty} w(t) w(t-\varepsilon) d t=0$. Since $\varepsilon$ is a fraction of the pulse width of $w(t)$, it is assumed that $\int_{-\infty}^{\infty} w(t-\varepsilon) w\left(t-T_{p}\right) d t \approx 0$. Then assuming detection of the first symbol, the expression of (5) can be rewritten as

$$
y_{m}^{(i)}=\sqrt{E_{x}} b_{0,0} \sum_{l=0}^{L-1}\left|\beta_{m}(l)\right|^{2} \Lambda^{(i)}(l)+\sum_{l=0}^{L-1} \beta_{m}(l) \eta_{m}^{(i)}(l)
$$

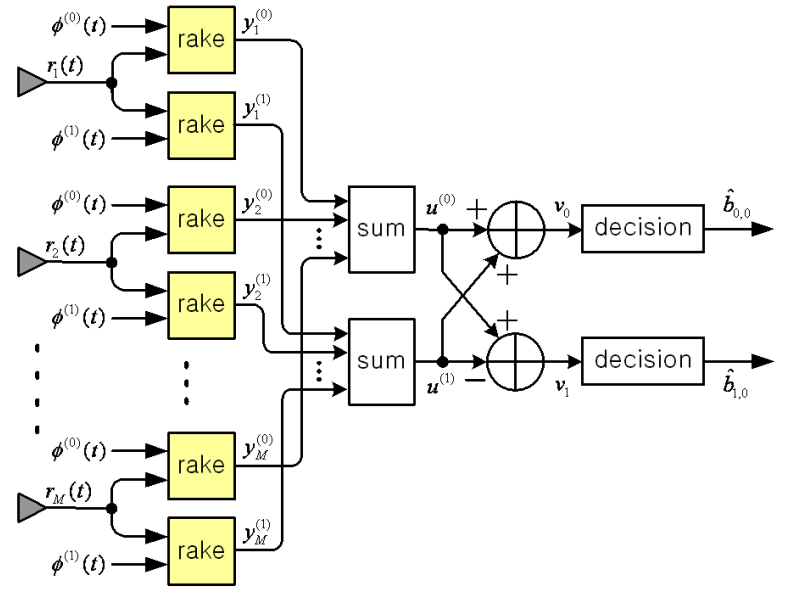

Fig. 1. Receiver architecture for the binary PAPM UWB receive diversity combining SIMO system.

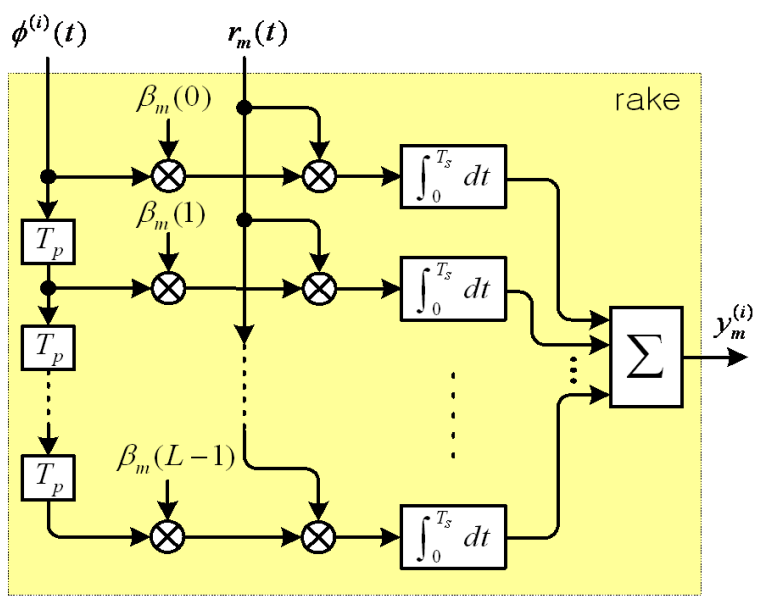

Fig. 2. Rake receiver with $L$ parallel correlators and time delay units at the $m$ th receive antenna.

where

$$
\begin{gathered}
\Lambda^{(i)}(l)=\int_{0}^{T_{s}} w\left(t-l T_{p}-\frac{1-b_{1,0}}{2} \varepsilon\right) w\left(t-l T_{p}-i \varepsilon\right) d t \\
\eta_{m}^{(i)}(l)=\int_{0}^{T_{s}} \eta_{m}(t) w\left(t-l T_{p}-i \varepsilon\right) d t
\end{gathered}
$$

In order to further exploit the receive diversity, the outputs of all $M$ rake receiver branches can be combined as

$$
u^{(i)}=\sum_{m=1}^{M} y_{m}^{(i)}
$$

Then the decision variables, $v_{0}$ and $v_{1}$, respectively, for the $b_{0,0}$ and $b_{1,0}$ detection can be given by 


$$
\begin{gathered}
v_{0}=u^{(0)}+u^{(1)} \\
=\sqrt{E_{x}} b_{0,0} \sum_{m=1}^{M} \sum_{l=0}^{L-1}\left|\beta_{m}(l)\right|^{2}\left(\Lambda^{(0)}(l)+\Lambda^{(1)}(l)\right) \\
+\sum_{m=1}^{M} \sum_{l=0}^{L-1} \beta_{m}(l)\left(\eta_{m}^{(0)}(l)+\eta_{m}^{(1)}(l)\right) \\
v_{1}=u^{(0)}-u^{(1)} \\
=\sqrt{E_{x}} b_{0,0} \sum_{m=1}^{M} \sum_{l=0}^{L-1}\left|\beta_{m}(l)\right|^{2}\left(\Lambda^{(0)}(l)-\Lambda^{(1)}(l)\right) \\
+\sum_{m=1}^{M} \sum_{l=0}^{L-1} \beta_{m}(l)\left(\eta_{m}^{(0)}(l)-\eta_{m}^{(1)}(l)\right)
\end{gathered}
$$

The decision variable for any bit of the transmitted data stream is expressed as $d_{k}=\operatorname{Re}\left\{v_{k}\right\}, k=0,1$. The bit decisions are made by independently passing $d_{k}$ through a decision device with a threshold of zero. The resulting instantaneous output signal to noise ratio (SNR) per bit, $\gamma_{b}$, is thus described as

$$
\gamma_{b}=\frac{E_{x}}{N_{o}} \sum_{m=1}^{M} \sum_{l=0}^{L-1}\left|\beta_{m}(l)\right|^{2}
$$

\section{TIME-SWITCHED TRANSMIT DIVERSITY APPROACH}

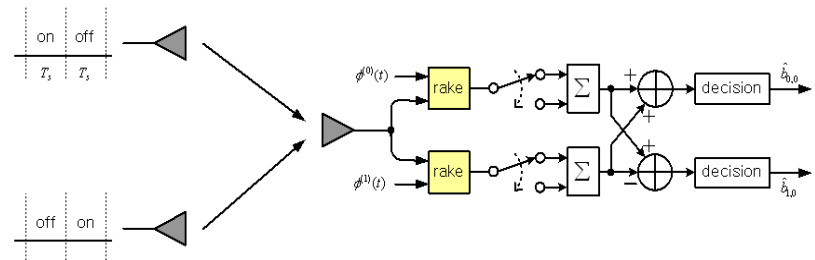

Fig. 3. bianry PAPM UWB TSTD MISO system with $N=2$.

Now briefly introduce a TSTD scheme in UWB MISO systems [4] for comparison with the receive diversity combining UWB systems with binary PAPM presented in section II. In this case, the same symbol is transmitted over the $N$ transmit antennas during $N$ symbol intervals as shown in Fig. 3. At each symbol interval, only one transmit antenna is turned on and the other antennas are tuned off. Performing MRC of the received signals during $N$ symbol times at one receive antenna creates the received SNR gain as follows:

$$
\gamma_{b}=\frac{E_{x}}{N_{o}} \sum_{n=1}^{N} \sum_{l=0}^{L-1}\left|\beta_{n}(l)\right|^{2}
$$

where $\beta_{n}(l)$ is the MISO channel fading coefficient of the $l$ th path for the signal to the receive antenna sent from the $n$th transmit antenna. When the number of transmit antennas in MISO systems is equivalent to the number of receive antennas in SIMO systems, the received SNR of (13) is the same as that of (12). Thus, the BER results for TSTD systems are identical to those of the receive diversity combining systems. The receiver of the TSTD system uses two rake combiners as shown Fig. 3 while the receive diversity SIMO system requires $2 M$ rake combiners in the receiver as shown in Fig. 1. That's why the TSTD system can offer less complexity in the receiver side. Note that even if the TSTD scheme yields an $N L$ fold diversity gain, it provides an inefficient utilization of the degrees of freedom available in the channel because it only transmits one symbol data per $N$ symbol times.

\section{SIMULATION RESULTS}

In the simulations of the receive diversity combining SIMO systems with binary PAPM, the SNR per symbol in decibel is defined as

$$
\zeta_{b}=\left(2 E_{x} / N_{0}\right) M \sum_{l=0}^{L-1} e^{-\rho l}
$$

For the simulations of the TSTD systems, $N$ replaces $M$ in the SNR definition of (14). The log-normal fading amplitude $\alpha_{m}(l)$ can be given by $\alpha_{m}(l)=e^{g_{m}(l)}$ with $g_{m}(l) \sim N\left(\mu_{g_{m}(l)}, \sigma_{g}^{2}\right)$ where the variance $\sigma_{g}^{2}$ is assumed to be independent of $m$ and $l$. The standard deviation of $20 \log _{10} \alpha_{m}(l)=g_{m}(l)\left(20 \log _{10} e\right)$ is assumed to be a 4-dB. The requirement for the average power of the $l$ th path to meet $E\left[\alpha_{m}(l)^{2}\right]=e^{-\rho l}$ is $\mu_{g_{m}(l)}=$ $-\sigma_{g}^{2}-\rho l / 2$ and $\sigma_{g}$ is given as $\sigma_{g}=4 /\left(20 \log _{10} e\right)$. Here, we use the power decay factor of $\rho=0.046$.

Figs. 4 and 5 show the BER performance of receive diversity combining SIMO and TSTD MISO UWB systems with binary PAPM as a function of SNR per bit $\zeta_{b}$ for different $M(N)$ and $L$ values, respectively. Spatial diversity using multiple receive antennas improves the BERs. Temporal diversity obtained by the augmentation of resolvable paths combined boosts the performance of SIMO and MISO systems. The multiple receive and transmit antennas can be used to reduce the number of resolvable multipaths combined while keeping the same error performance. It is shown that when $M=N$, the TSTD scheme in MISO systems achieve the same BER performance as that of receive diversity combining SIMO systems. It is found that as the number of transmit antennas increases, the TSTD MISO system enhances the performance. 


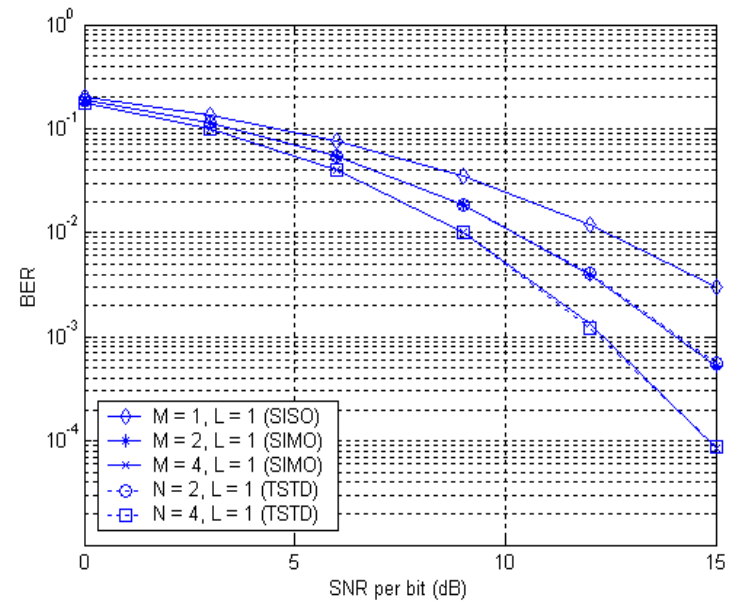

Fig. 4. BER versus SNR per symbol for receive diversity combining SIMO and TSTD MISO UWB systems for different $M(N)$ values.

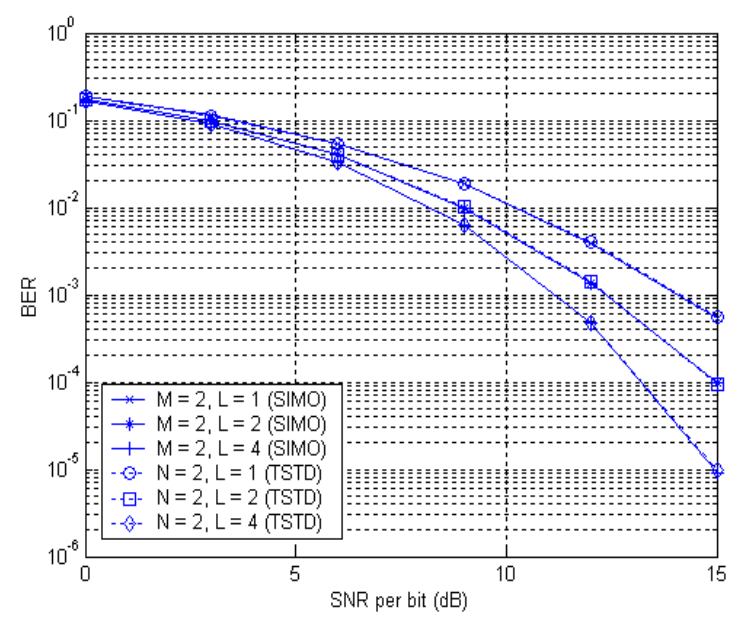

Fig. 5. BER versus SNR per symbol for receive diversity combining SIMO and TSTD MISO UWB systems for different $L$ values.

\section{CONCLUSIONS}

The error performance of UWB SIMO systems with binary PAPM based on the linear receive diversity has been presented over indoor log-normal multipath fading channels. It has been found that increasing the number of receive antennas improves the error performance of the rake diversity combining systems. It has been shown that the TSTD scheme in MISO systems offers the same performance as the receive diversity combining SIMO receiver. The TSTD system can reduce the computational load in the receiver side compared to the receive diversity combining receiver, but does not utilize efficiently the degrees of freedom available in the channel.

\section{ACKNOWLEDGMENT}

This study was supported by research funds from Dong-A University.

\section{REFERENCES}

[1] H. Liu, "High-Rate Transmission Scheme for Pulse-Based UltraWideband Systems Over Dense Multipath Indoor Channels," IEE Proc. Commun., vol. 152, no. 2, pp. 235-240, April 2005

[2] H. Liu, "Error Performance of a Pulse Amplitude and Position Modulated Ultra-Wideband System Over Lognormal Fading Channels," IEEE Commun. Letters, vol. 7, no. 11, pp. 531-533, Nov. 2003

[3] S. S. Tan, B. Kanna, and A. Nallanathan, "Performance of UWB Multiple-Access Impulse Radio Systems With Antenna Array in Dense Multipath Environments," IEEE Trans. Commun., vol. 54, no. 6, pp. 966-970, June 2006

[4] L. C. Wang, W. C. Liu, and K. J. Shieh, "On the Performance of Using Multiple Transmit and Receive Antennas in Pulse-Based Ultrawideband Systems," IEEE Trans. Wireless Commun., vol. 4, no. 6, pp. 2738-2750, Nov. 2005

[5] UWB Channel Modeling Contribution from Intel, IEEE P802.1502/279-SG3a.

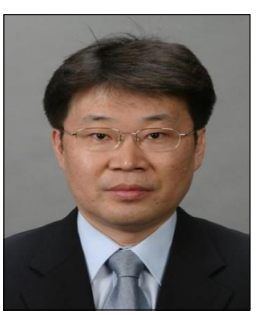

Sangchoon Kim was born in Jeju, Korea, in 1965. He received the B.S. degree from Yonsei University, Seoul, Korea, in electronics engineering in 1991 and the M.E. and Ph.D. degrees from the University of Florida, Gainesville, FL, all in electrical and computer engineering, in 1995 and 1999, respectively. From 2000 to 2005 , he had been a Research Engineer in LG Institute of Technology, Seoul and a Senior Research Engineer in LG Electronics, Anyang, Korea. Since 2005, he has been a faculty member in department of electronics engineering, Dong-A University, Busan, Korea. His research interests include synchronization, array signal processing techniques, and wireless mobile communication systems and algorithms including UWB, MIMO, OFDM, and WCDMA. 